\title{
Influences de l'éclairement et de la température sur divers paramètres de croissance de jeunes Ginkgo biloba L cultivés en conditions contrôlées de jours longs
}

\author{
V Flesch 1,2,3, M Jacques 2 *, L Cosson 3, V Pétiard 4, JP Balz 1 \\ 1 Institut H Beaufour, 17, avenue Descartes, 92350 Plessis-Robinson; \\ 2 Université Paris VI, laboratoire de physiologie de développement des plantes, \\ 4, place Jussieu, Tour 53, E5 75252 Paris cedex 05; \\ 3 Université Paris XI, laboratoire de botanique et de phytochimie, \\ avenue JB Clément, 92290 Châtenay-Malabry \\ 4 Francereco, 30-38, avenue G Eiffel, 37001 Tours, cedex, France
}

(Reçu le 9 juillet 1990; accepté le13 novembre 1990)

\begin{abstract}
Résumé - La croissance végétative de jeunes Ginkgo biloba $L$ a été étudiée en conditions contrôlées dans les serres et les enceintes climatisées du Phytotron à différentes températures et sous éclairements de longue durée $(16 \mathrm{~h})$ de qualité et de densité de flux quantique différentes. Cultivés en éclairement naturel ou en éclairement artificiel de $250 \mu \mathrm{E} \cdot \mathrm{m}^{-2} \cdot \mathrm{s}^{-1}$, à 24-17 ${ }^{\circ} \mathrm{C}$, les plants effectuent 1 ou 2 cycles végétatifs selon les individus (tableau l). Aux mêmes températures, un éclairement de $430 \mu \mathrm{E} \cdot \mathrm{m}^{-2} \cdot \mathrm{s}^{-1}$ favorise les reprises spontanées de croissance et permet à certains jeunes plants de réaliser 3 pousses. L'allongement caulinaire (fig 1), plus sensible aux variations de la lumière que l'émission foliaire (fig 2), est stimulé par un éclairement de $250 \mu \mathrm{E} \cdot \mathrm{m}^{-2} \cdot \mathrm{s}^{-1}$. Des températures élevées $\left(32-25^{\circ} \mathrm{C}\right)$ associées à un éclairement de $250 \mu \mathrm{E} . \mathrm{m}^{-2} . \mathrm{s}^{-1}$ maintiennent une majorité des plants en croissance active (fig $1 \mathrm{~A}$ ) pendant une longue durée.

Dans toutes les conditions (figs $3,4,5,6$ ), on retrouve une organisation périodique le long de l'axe principal (alternance de zones d'entrenœuds longs et courts). L'agencement spatial des entrenœuds n'est pas modifié par les facteurs de l'environnement, contrairement à l'agencement temporel accéléré aux températures élevées.

Le développement des bourgeons axillaires en auxiblastes ou en brachyblastes est toujours limité (tableaux II, III). II se produit au niveau des entrenœuds longs (figs $3,4,5,6$ ).
\end{abstract}

Ginkgo biloba / crolssance / densité de flux quantique / température / ramification

Summary - Effects of light and temperature on growth of Ginkgo blloba cultivated under controlled long day conditions. The vegetative growth of Ginkgo biloba $L$ (Ginkgoaceae) at varying temperatures and under different types of light (natural or artificial) has been described. Young Ginkgo plants originating from Korean seeds were grown in Phytotron controlled greenhouses at $24-17{ }^{\circ} \mathrm{C}, 16 \mathrm{~h}$ day length in small climate chambers at either $24-17^{\circ} \mathrm{C}$, with $16 \mathrm{~h}$ of 250 or 430 $\mu E . m^{2} . s^{-1}$ for 40 wk or at $32-35^{\circ} \mathrm{C}$, with $16 \mathrm{~h}$ of $250 \mu \mathrm{E} . \mathrm{m}^{-2} . \mathrm{s}^{-1}$ for 20 wk (due to the limited height of the climate chambers).

Table I showed the heterogenous growth of plants in relation to environmental conditions. At 24$17^{\circ} \mathrm{C}$, under natural light or moderate artificial light $\left(250 \mu \mathrm{E} \cdot \mathrm{m}^{-2} \cdot \mathrm{s}^{-1}\right)$, Ginkgo plants completed 1 or

* Correspondance et tirés à part 
2 growth cycles, corresponding to 1 or 2 shoots. Under high energy light $\left(430 \mu E . m^{2} . s^{-1}\right)$ at the same temperature, we observed 3 successive growth cycles for some plants (figs $1 \mathrm{C}$ and $2 \mathrm{C}$ ). At warm temperatures $\left(24-17^{\circ} \mathrm{C}\right)$, each growth period of a maximum duration of 10 wk ended in a rest period. At high temperatures $\left(32-25^{\circ} \mathrm{C}\right)$, the young Ginkgo plants were able to grow continuously for 20 wk. These temperatures enhanced both main stem elongation and young leaf emission on 1-shoot-plants (figs $1 A, 2 A$ ). At $24-17^{\circ} \mathrm{C}$, the main stem extension was enhanced by artificial light of $250 \mu E . m^{-2} . s^{-1}$ and inhibited by artificial light of $430 \mu E \cdot m^{-2} \cdot S^{-1}$ (figs $1 A, 1 B$ ). The production of leaves was less sensitive to quality and intensity of light than shoot growth. Plants with 1 shoot, cultivated under 3 variable light conditions at the same temperature produced the same number of leaves (fig 2A). Foliar emission was generally increased by an increase in temperature.

In all cases, the main axis presented a periodic organization with alternately long and short internodes (figs 3-6). The first shoot generally had 2 long internodal sections whereas the following shoots only had one. Environmental conditions did not modify the sequence (spatial organization) of these internodal areas, but could increase or reduce the number and size of the internodes constituting these areas. At $32-25{ }^{\circ} \mathrm{C}$, the plants presented more and longer internodes. High temperatures accelerated the arrangement of the internodal areas. At the 20th week, the profile of a young plant grown at 32$25^{\circ} \mathrm{C}$ was similar to the profile of a 2-shoot plant grown at 24-17 ${ }^{\circ} \mathrm{C}, 430 \mu E . m^{-2} .5^{-1}$. for 40 wk (fig 4, plant No 83; fig 6, plant No 186). After bursting, axillary buds of Gingko biloba gave out short or long shoots (figs 3-6). Long shoots only presented 1 long internodal section. Ramification was influenced by the spectral quality of light and temperature (tables II, III). Most plants growing under artificial illumination at $24-17^{\circ} \mathrm{C}$ or $32-25^{\circ} \mathrm{C}$ had short or long shoots.

Conversely, natural day light reduced bud reactivation and shoot development. Extension of long shoots, as well that of the main axis was enhanced by high temperatures.

Ginkgo biloba / growth / photon flux density / temperature / branching

\section{INTRODUCTION}

Le Ginkgo biloba L, ultime représentant de la famille des Ginkgoacées dont l'apogée remonte aux ères secondaire et tertiaire, cultivé d'abord en Chine, est aujourd'hui répandu dans la plupart des zones tempérées du globe. Durant la saison printanière, le Ginkgo effectue une première pousse, qui est parfois suivie d'une deuxième pousse ou "pousse d'août" pendant l'été. Ce phénomène de pousses multiples au cours d'une saison de végétation est fréquent chez les espèces ligneuses tempérées (Lavarenne-Allary, 1965). Cependant, les fluctuations saisonnières de lumière et de température existant dans les régions tempérées imposent une contrainte pour le développement végétatif des arbres en provoquant un arrêt total de croissance quand les conditions du milieu deviennent défavorables (températures fraîches, jours de courte durée). Les arbres tropicaux présentent un comportement semblable avec une ou plusieurs phases de croissance pendant la saison des pluies interrompues par l'arrivée de la saison sèche (Hallé et Martin, 1968; Alvim et Alvim, 1976; Scarrone, 1965). Cette capacité de réaliser des poussées successives pendant la saison favorable semble correspondre à l'extériorisation d'un rythme de croissance propre à chaque plant interrompu par l'occurence de conditions climatiques défavorables. Ceci a été démontré d'abord chez des espèces herbacées (Millet, 1970), puis pour des espèces ligneuses des régions tempérées (Lavarenne et al, 1971; Barnola et al, 1977; Nozeran et al, 1983) ou tropicales (Maillard et al, 1987a) cultivés en conditions artificielles. 
L'étude de la croissance de Ginkgo biloba a fait l'objet d'un certain nombre de travaux, dont ceux de Gunckel et Thimann (1949) et de Critchfield (1970) sur le déterminisme de la ramification. De Sloover (1961b, 1962) a, entre autres résultats, mis en évidence les effets favorables sur la croissance de plants de semis d'une photopériode longue, contrairement à une photopériode courte. Nos expérimentations sont par conséquent réalisées en conditions de jours longs (16 h).

Cet article présente l'étude cinétique de la croissance suivant 2 critères (allongement de laxe principal, émission des organes foliaires) et les données concernant la longueur finale des entrenœuds et les niveaux d'insertion des ramifications. Ainsi sont révélés les régimes de lumière et de température les plus favorables à la croissance et des potentialités de croissance, masquées en conditions naturelles.

\section{MATÉRIEL ET MÉTHODES}

\section{Matériel végétal et paramètres de croissance mesurés}

L'étude est effectuée sur des jeunes Ginkgo biloba issus de semences non sélectionnées en provenance de Corée, qui nous sont fournies par le laboratoire Beaufour.

Chaque expérience porte sur 10 plants. L'observation individuelle des plants est effectuée chaque semaine pendant 40 semaines $(20$ semaines seulement à $32-25^{\circ} \mathrm{C}$ en raison de la hauteur limitée de l'enceinte climatisée). L'allongement de l'axe principal est mesuré avec une précision de $\pm 1 \mathrm{~mm}$. Le nombre de feuilles émises est noté ainsi que le comportement des bourgeons axillaires. L'activité apparente du méristème apical est également caractérisée, qu'il soit en croissance en édifiant l'axe et produisant des feuilles visibles ou en repos apparent, entouré d'écailles vertes puis brunes. Nous définissons une pousse comme étant une période d'activité de croissance globale (tige et/ou feuilles) qui débute par le débourrement d'un bourgeon terminal et qui s'achève par la différenciation d'un nouveau bourgeon terminal écailleux. Au sein d'une même pousse, 2 périodes d'allongement caulinaire peuvent se succéder.

\section{Conditions de culture}

Après conservation à $4{ }^{\circ} \mathrm{C}$, les semences sont mises à germer dans des terrines contenant de la vermiculite, en serre à $22^{\circ} \mathrm{C}$ sous l'éclairement naturel complété à $16 \mathrm{~h}$ par un éclairement artificiel mixte (incandescent et fluorescent) de $125 \mu \mathrm{E} \cdot \mathrm{m}^{-2} \cdot \mathrm{s}^{-1}$. Les plantules sont repiquées en pots individuels $15 \mathrm{j}$ après l'émergence de l'hypocotyle et sont placées dans les diverses conditions expérimentales.

Les expériences sont réalisées en photopériode de $16 \mathrm{~h}$. Les facteurs externes dont nous nous proposons d'étudier l'effet sur la croissance du Ginkgo biloba sont la densité de flux quantique de l'éclairement et la température. Les conditions dont nous avons disposé au Phytotron de Gif/Yvette sont :

- 1 serre à $24-17^{\circ} \mathrm{C}$ aux mêmes conditions d'éclairement naturel et artificiel que celles de mise en germination,

- 2 enceintes climatisées à $24-17{ }^{\circ} \mathrm{C}$ et 32 $25^{\circ} \mathrm{C}$ avec un éclairement dit "phytotronique" de $250 \mu \mathrm{E} \cdot \mathrm{m}^{-2} \cdot \mathrm{s}^{-1}$ foumi par des tubes fluorescents (Mazda 65, T12, Blanc Industrie) et des lampes à incandescence;

- une enceinte climatisée à $24-17^{\circ} \mathrm{C}$ avec éclairement fort de $430 \mu \mathrm{E} . \mathrm{m}^{-2} \cdot \mathrm{s}^{-1}$ produit par des lampes à halogénures métalliques (OSRAM HQI, 3,5 kW).

Dans ces différentes conditions d'éclairement et de thermopériode, l'humidité relative de l'air est maintenue à $70 \%$. Les plants sont arrosés à l'eau désionisée 2 fois/j et reçoivent de la solution nutritive (de Bilderling et Lourtioux, 1976) 3 fois par semaine. 


\section{RÉSULTATS}

\section{Allongement caullnaire ot émission des feullles}

Aux températures tièdes $\left(24-17^{\circ} \mathrm{C}\right)$, sous éclairement naturel et sous éclairement artificiel de $250 \mu \mathrm{E} . \mathrm{m}^{-2} \cdot \mathrm{s}^{-1}$ (tableau I), les plants effectuent majoritairement une seule pousse d'une durée de 8,5 semaines (éclairement naturel) ou de $10 \mathrm{se}$ maines (éclairement phytotronique). Néanmoins, dans ces conditions, quelques Ginkgo sont capables de réaliser une deuxième pousse là la fois allongement de l'axe principal et émergence de nouvelles feuilles) d'une durée plus brève (4 semaines) que la précédente. Aux mêmes températures, la présence d'un éclairement plus intense $\left(430 \mu \mathrm{E} . \mathrm{m}^{-2} . \mathrm{s}^{-1}\right)$ permet à $1 / 3$ des individus de réaliser 3 pousses. La durée des 2 premières pousses effectuées en éclairement fort est similaire à celles des pousses effectuées en éclairement naturel. La durée de la $3^{\theta}$ pousse n'est pas connue car la croissance n'était pas achevée quand l'expérience fut interrompue.

Aux températures élevées $\left(32-25^{\circ} \mathrm{C}\right)$ sous éclairement phytotronique, à l'inverse des températures tièdes, la majeure partie des jeunes Ginkgo présente une seule phase de croissance globale, soit avec un arrêt apparent et formation d'un bourgeon écailleux à la $19^{\circ}$ semaine (60\% des cas), soit de façon continue pendant les 20 semaines d'observation ( $20 \%$ des cas). Les plants à croissance continue et les plants à 1 pousse présentent 2 périodes d'élongation intense (2 périodes de 9 semaines pour les plants à croissance continue, une première période de 11,7 semaines et une deuxième période de 4,8 semaines pour les plants à 1 pousse) et une seule période d'initiation et d'émission foliaire (pé- riode de plus de 20 semaines pour les plants à croissance continue et de 19 semaines pour les plants à 1 pousse).

La vitesse (pente de la courbe) et l'intensité de la croissance caulinaire varient selon les conditions d'environnement et le nombre de pousses effectuées (fig 1). En régime tempéré $\left(24-17^{\circ} \mathrm{C}\right)$, les plants monocycliques (fig 1A) cultivés sous éclairement artificiel modéré ou fort atteignent sensiblement la même taille. Sous éclairement naturel, la petite taille des plants à 1 pousse résulte de l'absence de la deuxième période d'élongation caulinaire intense caractéristique de la première pousse dans les autres conditions. La croissance caulinaire des plants qui effectuent 2 pousses (fig 1B) est 2 fois plus importante sous éclairement artificiel de 250 $\mu \mathrm{E} \cdot \mathrm{m}^{-2} \cdot \mathrm{s}^{-1}$ que dans les 2 autres conditions d'éclairement. Les plants à 3 pousses (fig $1 \mathrm{C}$ ) que l'on ne rencontre que sous fort éclairement présentent des périodes d'allongement brèves et très intenses. On

Tableau I. Répartition des plants de jeunes Gingko biloba selon leurs modalités de croissance en fonction de la température, de la qualité et de la densité de flux quantique de l'éclairement. Dix plants par condition expérimentale.

Conditions

Pourcentage de plants effectuant expérimentales $1 P^{\circ} \quad 2 P^{\circ} \quad 3 P^{\circ}$ $\mathrm{CC}^{\circ}$

$\begin{array}{lllll}24-17^{\circ} \mathrm{C} \mathrm{en} & 70 & 30 & & \\ 24-17^{\circ} \mathrm{C} \mathrm{ep} & 70 & 30 & & \\ 24-17^{\circ} \mathrm{C} \mathrm{EF} * & 30 & 40 & 30 & \\ 32-25^{\circ} \mathrm{C} \mathrm{ep} \text { ep }^{*} & 60 & 20 & & 20\end{array}$

* en éclairement naturel, ep : éclairement de type phytotron $\left(250 \mu \mathrm{E} \cdot \mathrm{m}^{-2} \cdot \mathrm{s}^{-1}\right)$, EF : éclairement fort (430 $\left.\mu \mathrm{E} \cdot \mathrm{m}^{-2} \cdot \mathrm{s}^{-1}\right) ;{ }^{\circ} 1 \mathrm{P}$ : plants à 1 pousse, $2 \mathrm{P}$ : plants a 2 pousses, 3P : plants à 3 pousses, $C C$ : plants a croissance continue; absence de valeur : absence de pousse correspondante. 
constate que la période de repos succédant à la deuxième pousse est plus longue que celle qui se produit après la première pousse (13 semaines contre $10 \mathrm{se}$ maines). A $32-25^{\circ} \mathrm{C}$, les plants mono- ou bicycliques ou en croissance continue (fig $1 \mathrm{~A}$ et $1 \mathrm{~B})$ présentent une première période de croissance caulinaire réduite suivie d'une deuxième période de croissance

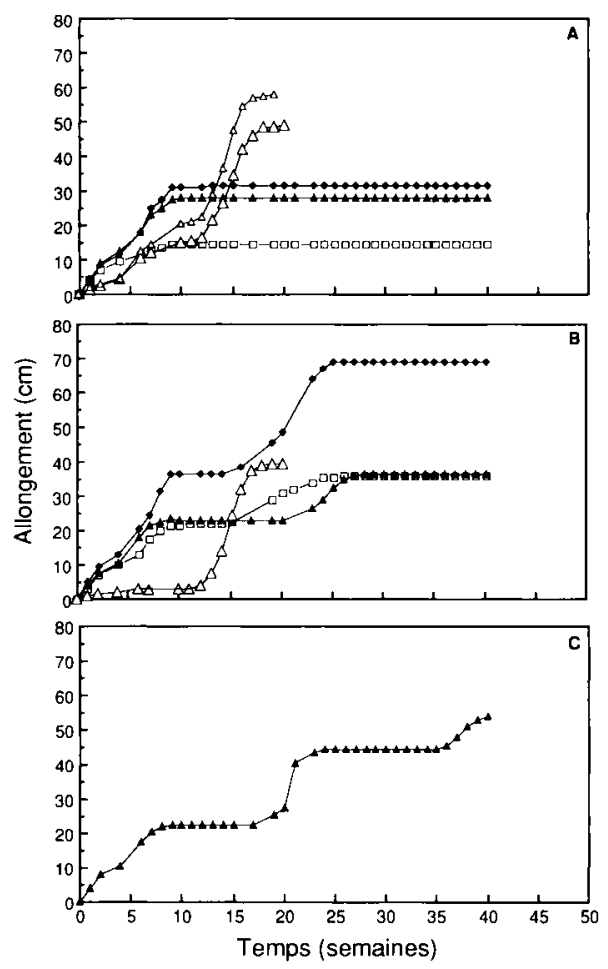

Fig 1. Influence de la température, de la qualité et de la densité de flux quantique de l'éclairement sur l'allongement de l'axe principal de jeunes Ginkgo biloba cultivés en $16 \mathrm{~h}$ à $32-25^{\circ} \mathrm{C}$, éclairement de type phytotron, $250 \mu \mathrm{E} \cdot \mathrm{m}^{-2} \cdot \mathrm{s}^{-1}(\Delta: 1$ pousse ou $\Delta$ : croissance continue), et à $24-17{ }^{\circ} \mathrm{C}$, éclairement naturel (a), éclairement de type phytotron, $250 \mu \mathrm{E} \cdot \mathrm{m}^{-2} \cdot \mathrm{s}^{-1}(\bullet)$ et éclairement fort, $430 \mu \mathrm{E} . \mathrm{m}^{-2} \cdot \mathrm{s}^{-1}(\Delta)$, selon le nombre de pousses effectuées. A: 1 pousse et croissance continue, B: 2 pousses, C: 3 pousses. Chaque point $=$ moyenne des valeurs de 10 plants. d'une vitesse et d'une intensité exceptionnelles. Les plants à croissance continue ont un axe principal légèrement plus grand que celui des plants à 1 pousse. C'est, avec l'absence d'arrêt de croissance et de différenciation d'un bourgeon terminal écailleux, la seule différence entre ces 2 types de plants.

Les écarts à la moyenne des valeurs d'allongement caulinaire ont des valeurs élevées et ne sont donc pas figurés sur les courbes. Ils varient de façon très importante selon le nombre de pousses qu'effectuent les plants. Le coefficient de variation de l'allongement réalisé par les plants à une pousse cultivés à $24-17{ }^{\circ} \mathrm{C}$ est assez faible, de l'ordre de 10-20\%, alors qu'il est de $30-40 \%$, sa valeur maximale pouvant atteindre $70 \%$, chez les plants à 2-3 pousses dans les mêmes conditions de thermoperiode. Ces différences sont la conséquence d'une plus grande homogénéité des plants à 1 pousse qui s'allongent peu et de façon synchronisée, alors que les phases de croissance des plants à pousses multiples sont à la fois désynchronisées et très intenses, creusant ainsi des écarts importants entre les plants. Ce phénomène est encore plus marqué à $32-25^{\circ} \mathrm{C}$, condition qui permet d'obtenir une croissance exceptionnelle, tant pour la durée, que pour l'intensité de la phase d'élongation caulinaire (coefficient de variation de l'allongement de l'ordre de $30 \%$ pour les plants à 1-2 pousses, sa valeur maximale pouvant atteindre $110 \%$ ).

Dans toutes les conditions expérimentales, on observe que la phase d'émission foliaire dure 3-7 j de plus que la phase d'allongement de l'axe principal. En effet, au début d'une nouvelle pousse, les feuilles préformées à l'intérieur du bourgeon terminal à la fin de la pousse précédente émergent avant que l'axe principal n'ait repris sa croissance (Critchfield, 
1970). À la fin d'une pousse, il se forme très fréquemment une rosette terminale de feuilles, séparées par des entrencuds de longueur très faible. Contrairement à l'allongement caulinaire, l'émission de feuilles (fig 2A, B et C) est peu sensible à la nature et la valeur de l'éclairement. Ainsi, pour les plants effectuant une seule pousse, à $24-17^{\circ} \mathrm{C}$, le nombre de feuilles émises et plastochrone apparent sont identiques en éclairement naturel ou en éclairement artificiel fort ou modéré. Quand une deuxième pousse se réalise, un fort niveau d'éclairement $\left(430 \mu \mathrm{E} \cdot \mathrm{m}^{-2} \cdot \mathrm{s}^{-1}\right)$ semble défavorable à l'activité d'organogenèse foliaire alors que des éclairements naturel ou artificiel de type phytotron permettent un dégagement presque permanent de nouvelles feuilles. Les conditions de températures élevées stimulent l'initiation et l'émergence de feuilles nouvelles. En effet, pour les plants à 1 pousse ou en croissance permanente, le nombre de feuilles formées est identique et toujours très supérieur à ceux des plants à 1 pousse cultivés à $24-17^{\circ} \mathrm{C}$. En revanche, les plants à 2 pousses portent le même nombre de feuilles à $32-25{ }^{\circ} \mathrm{C}$ et à $24-$ $17^{\circ} \mathrm{C}$, en ou ep. Toutefois, cela représente un nombre considérable de feuilles émergées à $32-25^{\circ} \mathrm{C}$ proportionnellement à la petite taille des plants dans ces conditions.

\section{Longueur des entrenœuds}

Le profil de la première pousse des Ginkgo mono- ou pluricycliques croissant en conditions d'éclairement artificiel, à 24$17^{\circ} \mathrm{C}$ et à $32-25{ }^{\circ} \mathrm{C}$ est constitué par la succession de 2 vagues d'entrenœuds longs séparées par une zone d'entrenœuds courts (figs 3,4 et 5). II s'achève par une série plus ou moins importante, selon les individus, d'entrenœuds courts.
La formation d'entrenœuds courts est à mettre en relation avec le ralentissement de l'élongation caulinaire observé vers la $5^{\theta}$ ou la $6^{\theta}$ semaine. En éclairement naturel, la deuxième vague de croissance de la première pousse comporte le plus souvent des entrenœuds courts (fig 6).
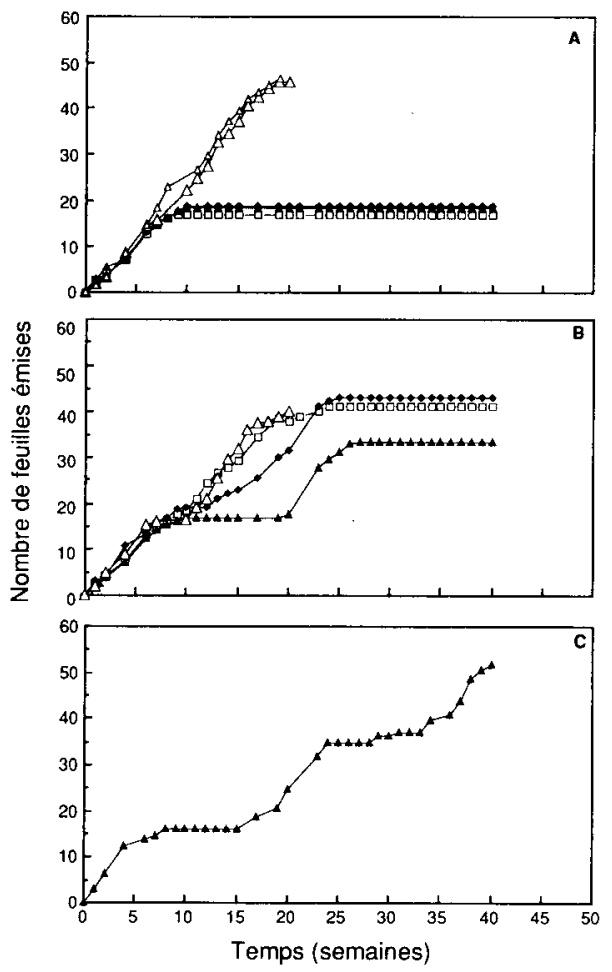

Fig 2. Influence de la température, de la qualité et de la densité de flux quantique de l'éclairement sur le nombre de feuilles émises par de jeunes Ginkgo biloba cultivés en 16 h à 32$25^{\circ} \mathrm{C}$, éclairement de type phytotron, $250 \mu \mathrm{E} \cdot \mathrm{m}^{-2} \cdot \mathrm{s}^{-1}(\Delta: 1$ pousse ou $\Delta$ : croissance continue), et à $24-17^{\circ} \mathrm{C}$, éclairement naturel (D), éclairement de type phytotron, $250 \mu \mathrm{E} . \mathrm{m}^{-}$ ${ }^{2} . s^{-1}(\diamond)$ et éclairement fort, $430 \mu \mathrm{E} \cdot \mathrm{m}^{-2} \cdot \mathrm{s}^{-1}(\Delta)$, selon le nombre de pousses effectuées. A: 1 pousse et croissance continue, B: 2 pousses, $C$ : 3 pousses. Chaque point $=$ moyenne des valeurs de 10 plants. 


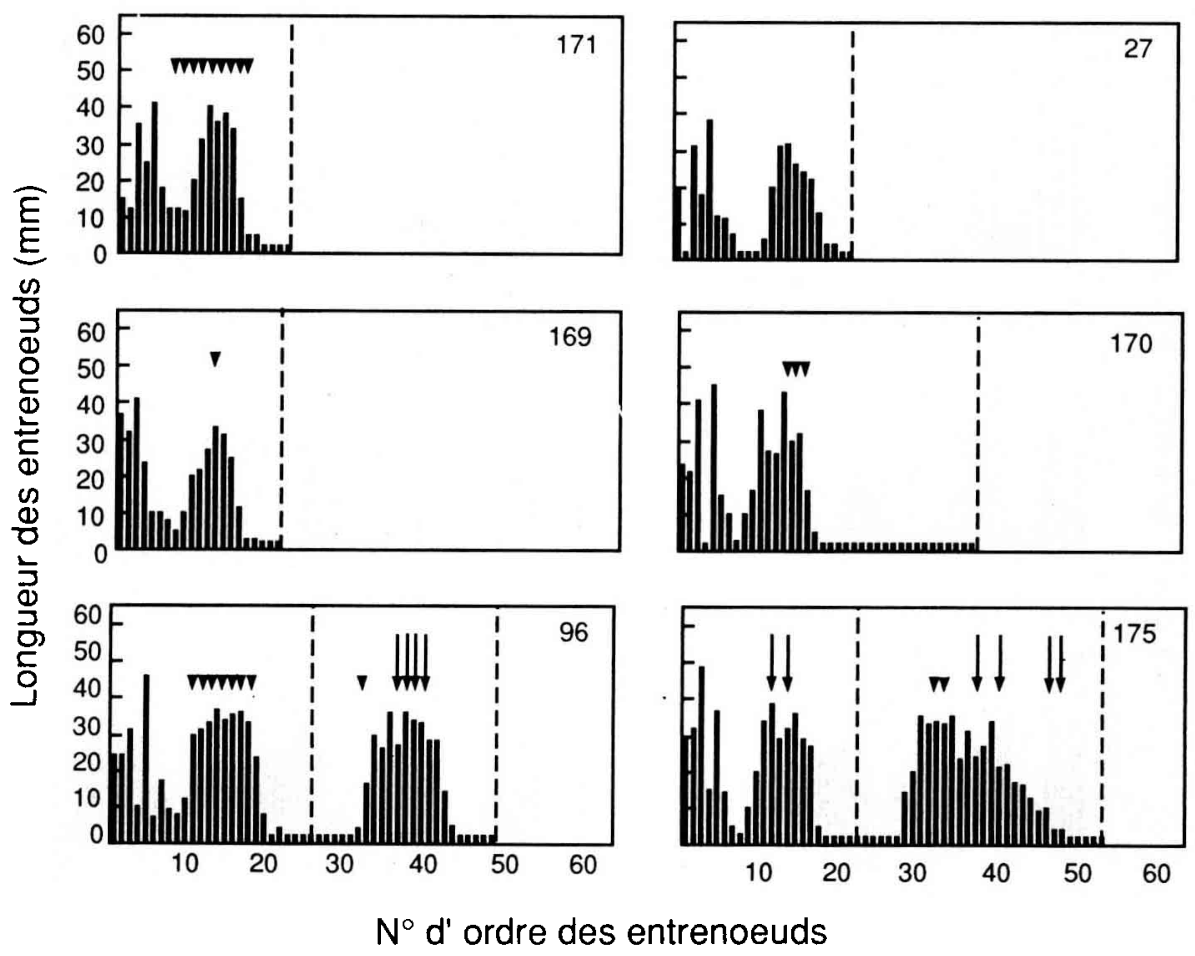

Fig 3. Longueurs finales des entrencuds et niveau d'insertion des brachyblastes $(\nabla)$ et des auxiblastes $(\downarrow)$ sur des Ginkgo biloba âgés de 10 mois et cultivés en enceinte climatisée à $24-17^{\circ} \mathrm{C}, 16 \mathrm{~h}$, $250 \mu \mathrm{E} \cdot \mathrm{m}^{-2} \cdot \mathrm{s}^{-1}$. Chaque graphique est le profil d'une plante, identifiée par un numéro.

En enceinte climatisée, quelle que soit la thermopériode, la deuxième pousse est constituée d'une vague d'entrenceuds courts correspondant à la simple émergence de feuilles préformées dans le bourgeon et d'une vague d'entrenœuds de grande dimension et en nombre important (de 11 à 20 selon les individus), responsables de l'extension de l'axe caulinaire néoformé. Elle s'achève par la production d'une rosette de feuilles que séparent des entrenœuds courts ou nuls. Le profil de la $3^{e}$ phase de croissance effectuée par certains plants sous fort éclairement est, soit identique à celui de la $2^{e}$ pousse, soit ré- duit à une succession d'entrenœuds très courts.

Le rythme temporel d'alternance entrenceuds longs et courts est tributaire des conditions du milieu, alors que le rythme spatial des entrenœuds semble prédéterminé pour l'espèce. En effet, à $32-25^{\circ} \mathrm{C}$, les plants effectuant une pousse ou en croissance continue peuvent présenter à la $20^{\circ}$ semaine la même organisation spatiale que les plants à 2 pousses après 40 semaines de culture à $24-17{ }^{\circ} \mathrm{C}$, sous éclairement fort ou modéré (fig 4, plant 83; fig 5, plant 186). 


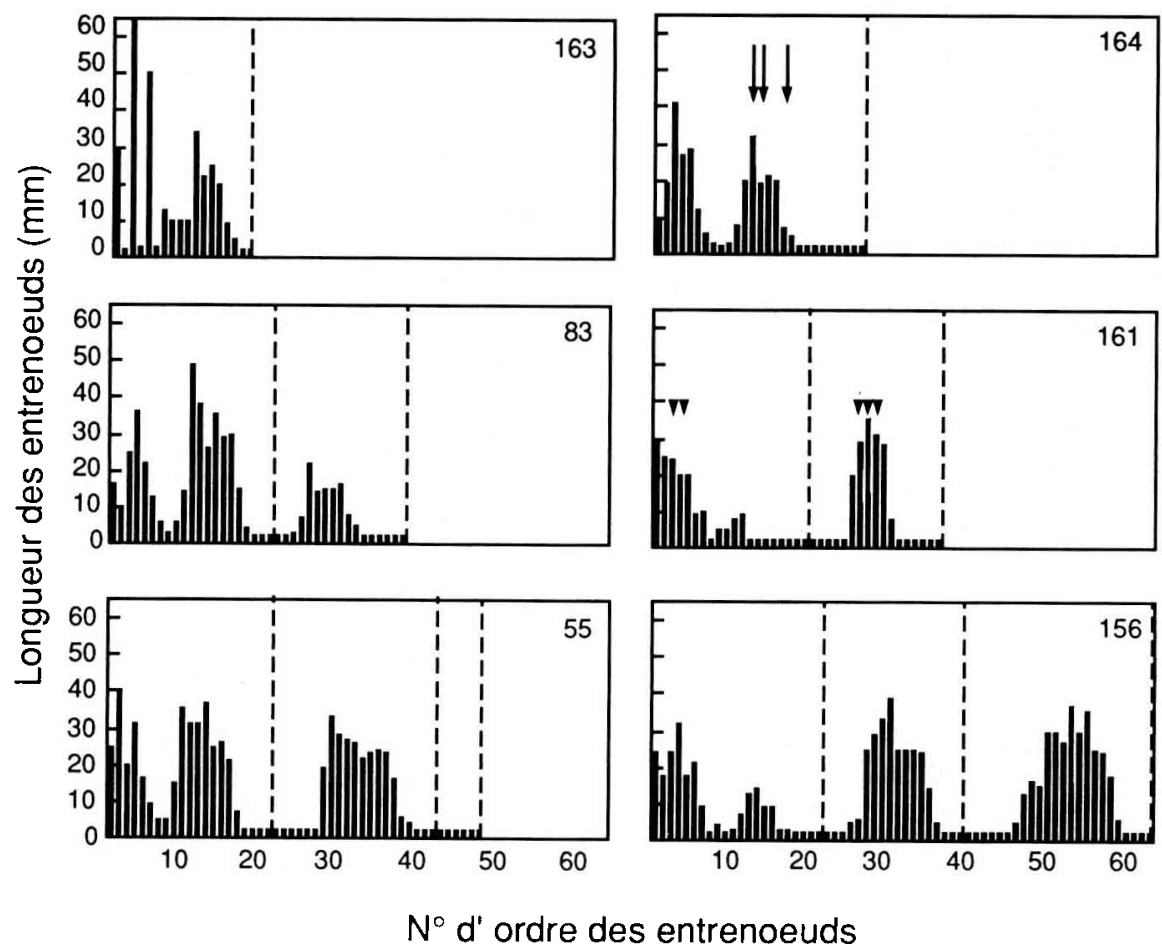

Fig 4. Longueurs finales des entrenœuds et niveau d'insertion des brachyblastes $(\nabla)$ et des auxiblastes ( $\downarrow$ ) sur des Ginkgo biloba âgés de 10 mois et cultivés en enceinte climatisée à $24-17^{\circ} \mathrm{C}, 16$ $h, 430 \mu E \cdot m^{-2} \cdot s^{-1}$. Chaque graphique est le profil d'une plante, identifiée par un numéro.

\section{Développement des bourgeons axillaires}

Les bourgeons axillaires entrent en végétation chez les plants de grande taille (qualifiés de vigoureux) que ces derniers soient mono- ou pluricycliques (figs $3,4,5$ et 6). Les débourrements se produisent par vagues successives au moment des ralentissements de l'élongation caulinaire. Ils sont localisés au niveau des entrenœuds longs. Les bourgeons évoluent sous forme de rosettes de feuilles (brachyblastes) ou de rameaux longs (auxiblastes). Le nombre de plants portant des brachyblastes (tableau II) est toujours très nettement supérieur au nombre de plants portant des auxiblastes dans toutes les conditions expérimentales. Les éclairements artificiels constants sont plus favorables à la réactivation des bourgeons axillaires en brachyblastes ou auxiblastes que l'éclairement naturel. Mais il faut remarquer que la valeur du flux quantique de l'éclairement artificel ne joue ici aucun rôle.

On note plus fréquemment l'apparition de rameaux longs sur les plants à pousses multiples que sur les plants ne réalisant qu'une pousse, sauf à $24-17{ }^{\circ} \mathrm{C}$, sous éclairement fort et à $32-25{ }^{\circ} \mathrm{C}$, sous éclai- 

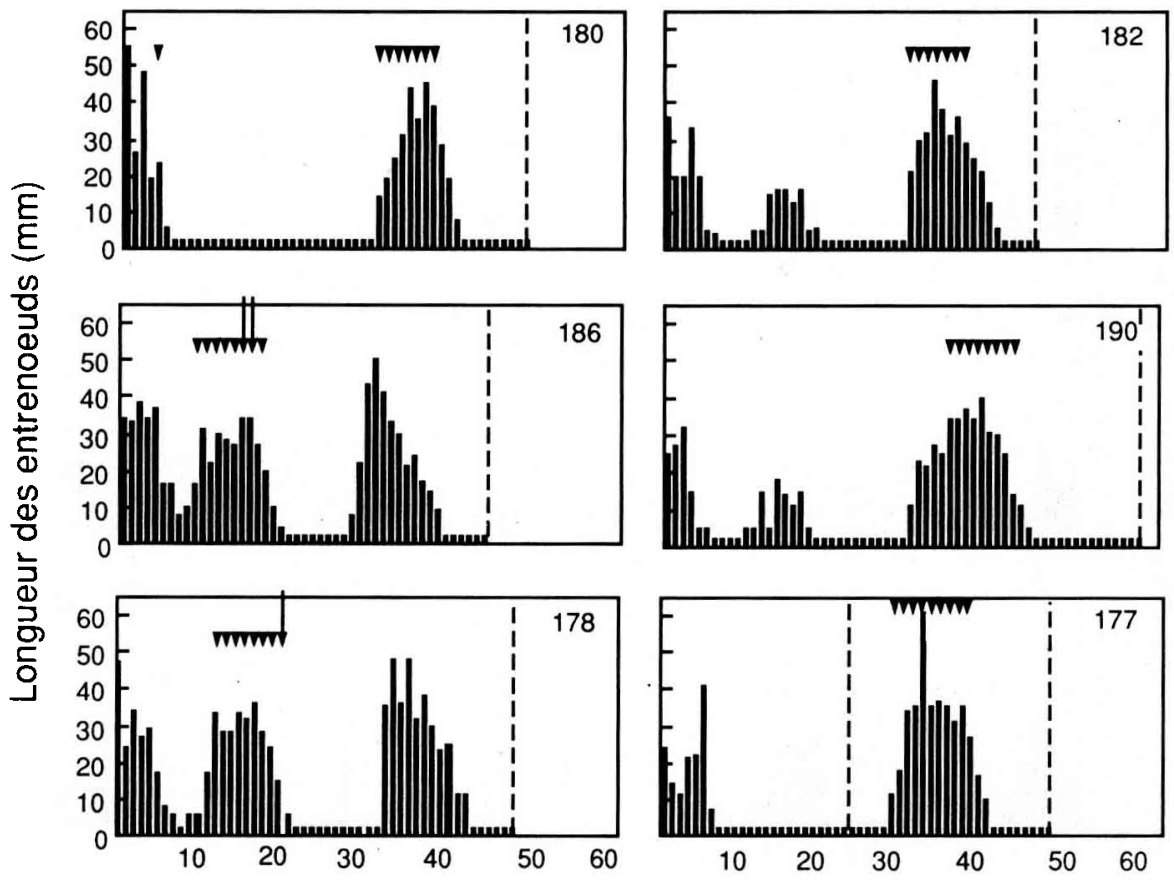

\section{$N^{\circ} d^{\prime}$ ordre des entrenoeuds}

Fig 5. Longueurs finales des entrenœuds et niveau d'insertion des brachyblastes ( $\nabla$ ) et des auxiblastes $(\downarrow)$ sur des Ginkgo biloba âgés de 10 mois et cultivés en enceinte climatisée à $32-25^{\circ} \mathrm{C}, 16 \mathrm{~h}$, $250 \mu \mathrm{E} \cdot \mathrm{m}^{-2} \cdot \mathrm{s}^{-1}$. Chaque graphique est le profil d'une plante, identifiée par un numéro.

rement phytotronique où seules les plants monocycliques portent des rameaux longs.

Le nombre de rosettes par plant (tableau III) et la quantité de feuilles par rosette augmentent avec le nombre de phases de croissance effectuées. II en est de même pour le nombre toujours restreint d'auxiblastes en serre et sous éclairement de type phytotron, à $24-17^{\circ} \mathrm{C}$. Une densité de flux quantique importante et surtout les températures élevées provoquent un allongement considérable des rameaux formés (longueur moyenne des auxiblastes portés par des plants monocycliques à $24-17^{\circ} \mathrm{C}$, éclairement fort : $10,3 \mathrm{~cm}$; à $32-25{ }^{\circ} \mathrm{C}$, éclairement phytotronique : $31,0 \mathrm{~cm}$; à 24$17^{\circ} \mathrm{C}$, éclairement phytotronique : $8,1 \mathrm{~cm}$, à $24-17{ }^{\circ} \mathrm{C}$, éclairement naturel : aucun auxiblaste formé). On retrouve l'effet stimulant des températures chaudes sur l'émission foliaire. En effet, on peut compter en moyenne 22 feuilles par auxiblaste à 32 $25^{\circ} \mathrm{C}$, éclairement phytotronique pour seulement 10,7 feuilles par auxiblaste à 24$17^{\circ} \mathrm{C}$, toutes natures et énergies d'éclairement confondues. 


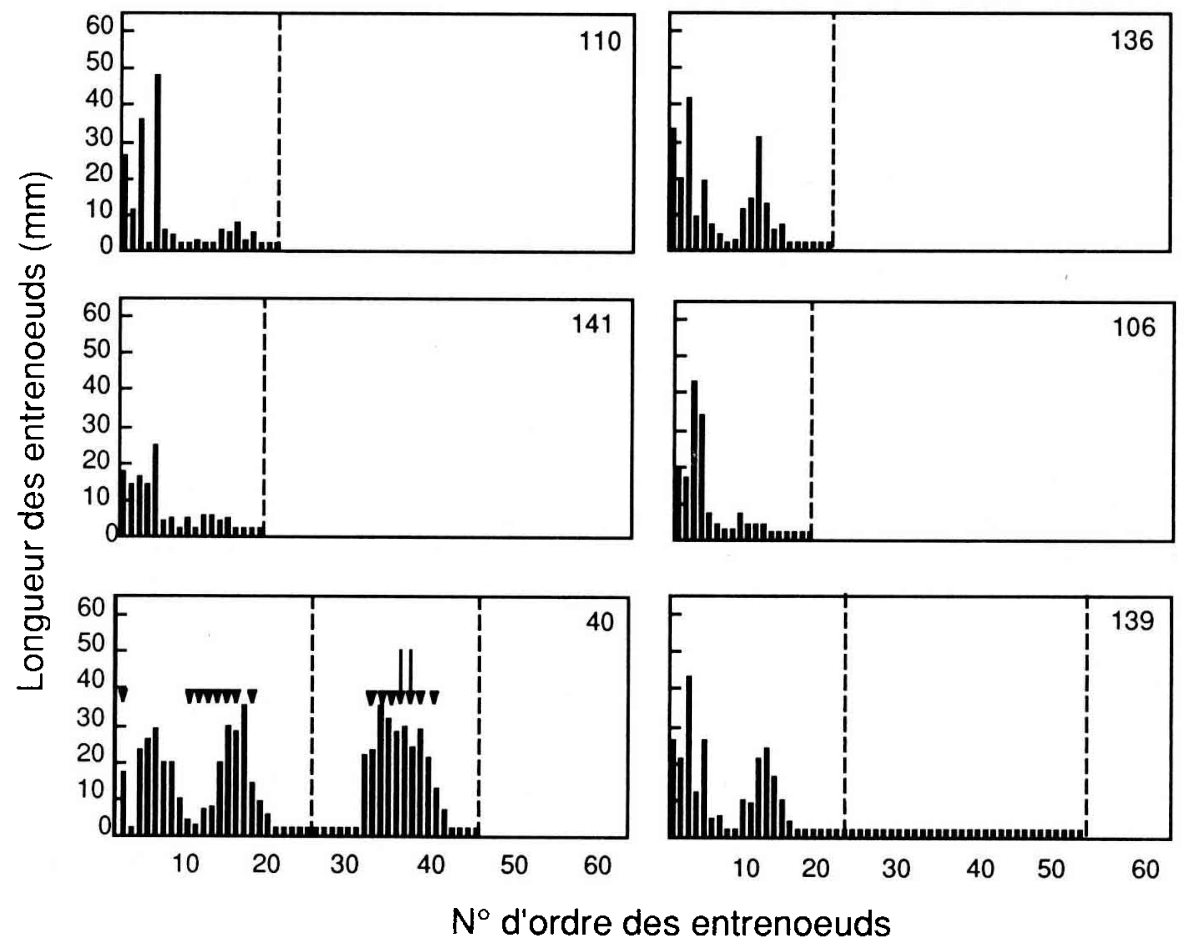

Fig 6. Longueurs finales des entrenœuds et niveau d'insertion des brachyblastes $(\nabla)$ et des auxiblastes $(\downarrow)$ sur des Ginkgo biloba âgés de 10 mois et cultivés en serre à $24-17^{\circ} \mathrm{C}, 16 \mathrm{~h}$. Chaque graphique est le profil d'une plante, identifiée par un numéro.

Tableau II. Influence de la température, de la qualité et de la densité de flux quantique de l'éclairement sur le pourcentage de plants ramifiés en fonction du nombre de pousses effectuées par de jeunes Ginkgo biloba. Dix plants par condition expérimentale.

\begin{tabular}{|c|c|c|c|c|c|c|c|c|c|c|}
\hline \multirow{2}{*}{$\begin{array}{c}\text { Conditions } \\
\text { expérimentales }\end{array}$} & \multicolumn{4}{|c|}{ Plants à brachyblastes } & \multicolumn{6}{|c|}{ Plants à auxiblastes } \\
\hline & \%total & $\% 1 P^{\circ}$ & $\% 2 P^{\circ}$ & $\% 3 P^{\circ}$ & $\% C C^{\circ}$ & \%total & $\% 1 P^{\circ}$ & $\% 2 P^{\circ}$ & $\% 3 P^{\circ}$ & $\% \mathrm{CC}^{\circ}$ \\
\hline $\begin{array}{l}24-17^{\circ} \mathrm{C} \mathrm{en} \\
24-17^{\circ} \mathrm{C} \mathrm{ep} \\
24-17^{\circ} \mathrm{C} \mathrm{EF} \\
32-25^{\circ} \mathrm{C} \mathrm{ep} \\
\text { ep }^{*}\end{array}$ & $\begin{array}{r}30 \\
90 \\
80 \\
100\end{array}$ & $\begin{array}{r}28 \\
86 \\
67 \\
100\end{array}$ & $\begin{array}{r}33 \\
100 \\
75 \\
100\end{array}$ & 100 & 100 & $\begin{array}{l}10 \\
40 \\
20 \\
30\end{array}$ & $\begin{array}{r}0 \\
14 \\
67 \\
33\end{array}$ & $\begin{array}{r}33 \\
100 \\
0 \\
0\end{array}$ & 0 & 50 \\
\hline
\end{tabular}

" en éclairement naturel, ep : éclairement de type phytotron $\left(250 \mu \mathrm{E} \cdot \mathrm{m}^{-2} \cdot \mathrm{s}^{-1}\right)$, EF : éclairement fort $\left(430 \mu \mathrm{E} \cdot \mathrm{m}^{-2} \cdot \mathrm{s}^{-1}\right)$; ${ }^{\circ} 1 \mathrm{P}$ : plants à 1 poussée, $2 \mathrm{P}$ : plants à 2 pousses, $3 \mathrm{P}$ : plants à 3 pousses, $\mathrm{CC}:$ plants a croissance continue; absence de valeur : absence de pousse correspondante. 
Tableau III. Influence de la température, de la qualité et de la densité de flux quantique de l'éclairement sur la ramification de jeunes Ginkgo biloba. Dix plants par condition expérimentale.

\begin{tabular}{|c|c|c|c|c|c|c|c|c|}
\hline \multirow{2}{*}{$\begin{array}{c}\text { Conditions } \\
\text { experimentales }\end{array}$} & \multicolumn{4}{|c|}{ Nombre de brachyblastes par plant } & \multicolumn{4}{|c|}{ Nombre d'auxiblastes par plant } \\
\hline & $1 P^{\circ}$ & $2 P^{\circ}$ & $3 P^{\circ}$ & $C C^{\circ}$ & $1 P^{\circ}$ & $2 P^{\circ}$ & $3 P$ & $C C^{\circ}$ \\
\hline $24-17^{\circ} \mathrm{C} \mathrm{en} *$ & 2 & 13 & 0 & & 0 & 1 & & \\
\hline $24-17^{\circ} \mathrm{C} \theta \mathrm{p}^{*}$ & 2,8 & 7,7 & & & 0,1 & 4 & & \\
\hline $24-17^{\circ} \mathrm{C} \mathrm{EF}$ & 0,7 & 5,2 & 8,3 & & 1 & 0 & 0 & \\
\hline $32-25^{\circ} \mathrm{C}$ ep $*$ & 9,7 & 8 & & 12,5 & 0,7 & 0 & & 0,5 \\
\hline
\end{tabular}

- en éclairement naturel, ep : éclairement de type phytotron $\left(250 \mu \mathrm{E} \cdot \mathrm{m}^{-2} \cdot \mathrm{s}^{-1}\right)$, EF : éclairement fort $\left(430 \mu \mathrm{E} \cdot \mathrm{m}^{-2} . \mathrm{s}^{-1}\right)$; -1P : plants à 1 poussée, $2 \mathrm{P}$ : plants à 2 pousses, $3 \mathrm{P}$ : plants à 3 pousses, $\mathrm{CC}$ : plants à croissance continue; absence de valeur : absence de pousse correspondante.

\section{DISCUSSION ET CONCLUSION}

Compte tenu du matériel végétal utilisé (semences non sélectionnées) et des observations effectuées par De Sloover (1961a, 1961b, 1962) sur une population issue de semences, le polymorphisme des jeunes Ginkgo était attendu. II est la résultante d'un mode de fonctionnement du méristème apical variable selon les individus. En conditions naturelles, la variabilité morphologique ne peut s'exprimer du fait d'une période propice à la croissance trop courte pour permettre aux plants d'effectuer plus d' 1 ou 2 pousses par an. Ce sont les photopériodes courtes de l'automne et de l'hiver plus que les températures fraîchissantes qui interdisent dans un premier temps tout maintien d'activité de croissance. En effet, De Sloover (1961b) a montré qu'une photopériode de $12 \mathrm{~h}$ bloque rapidement la croissance des jeunes semis maintenus à température tiède. Des photopériodes plus longues (14 ou $16 \mathrm{~h}$ ) prolongent l'activité apicale, lors de la première pousse et permettent une reprise d'activité dans le bourgeon terminal et donc une deuxième pousse pour une partie des plants.
Nos résultats sur les pourcentages de plants effectuant 1 ou 2 pousses en serre divergent notablement de ceux de $\mathrm{De}$ Sloover. Une différence d'évaluation des phases de croissance et de leurs limites est sans doute la source de ces différences. De Sloover considère probablement comme 2 pousses distinctes ce que sont pour nous les 2 périodes d'élongation caulinaire de la première pousse. Cela pourrait expliquer son recensement de quelques plants tricycliques en condition de serre, alors que nous n'observions que des plants mono- et bicycliques dans les mêmes conditions.

Les conditions de jours longs (16 h) et de température diurne tiède (au minimum égale à $22^{\circ} \mathrm{C}$ ) dans lesquelles nous avons placé les plants renforcent l'expression de leur polymorphisme en prolongeant les capacités de croissance. L'utilisation de températures élevées $\left(32-25^{\circ} \mathrm{C}\right)$ nous a permis d'observer l'aptitude du Ginkgo à croître en permanence, ce que les expériences, à température plus basse et de courte durée, réalisées par De Sloover n'avaient pu déceler. Chez le bouleau, Lavarenne et al (1971) obtiennent une croissance continue par la seule élévation de la 
température nocturne $\left(25{ }^{\circ} \mathrm{C}\right.$ constant contre $\left.25-12{ }^{\circ} \mathrm{C}\right)$. A température chaude, c'est non seulement le rythme de croissance du Ginkgo qui est modifié, mais également le nombre d'unités morphogénétiques et l'intensité de leur développement qui sont augmentés si on les compare à ceux obtenus à des températures tièdes pour un même éclairement. Cela peut sembler normal pour des espèces tropicales comme le Terminalia superba (Maillard et al, 1987a) ou le bananier (Turner et Lahav, 1983), mais est plus étonnant pour une espèce des régions tempérées pour laquelle les fortes températures freinent en général la morphogenèse et l'accroissement des organes formés (Juntilla, 1980; Morgan et al, 1985).

L'éclairement constant et de flux quantique moyen d'une enceinte climatisée modifie peu le rythme de croissance des jeunes Ginkgo, comparativement à celui existant sous éclairement naturel; dans les 2 cas, l'activité apicale ne se maintient pas au-delà de 2 cycles végétatifs, à la différence d'un éclairement intense et constant de $430 \mu \mathrm{E} \cdot \mathrm{m}^{-2} \cdot \mathrm{s}^{-1}$ qui autorise la réalisation de 3 pousses successives. Mais un fort niveau d'éclairement artificel limite l'élongation de l'axe principal et le plastochrone apparent, alors qu'un éclairement artificiel de moyenne énergie induit une croissance particulièrement importante du Ginkgo. Warrington et al (1978) ainsi que Morgan et al (1985) ont démontré qu'un flux de photons trop important pouvait se révéler inhibiteur de l'allongement des entrenœuds et de la production de feuilles. Chez Ginkgo biloba, il est intéressant de constater que l'effet inhibiteur du fort éclajrement s'observe surtout sur les deuxième et troisième pousses et assez peu sur la première pousse.

Certains critères de croissance des jeunes Ginkgo tels que la vitesse d'allongement et la longueur finale de l'axe princi- pal, subordonnées à la croissance des entrencuds, ainsi que le plastochrone et le nombre de feuilles émises, sont influencés par les facteurs de l'environnement. D'autres, tels que l'alternance entrenceuds longs - entrenœuds courts et le niveau d'insertion des ramifications en sont indépendants. Ainsi, l'alternance périodique des zones d'entrenœuds longs et d'entrenœuds courts est identique à $24-17^{\circ} \mathrm{C}$ et à $32-25^{\circ} \mathrm{C}$. Le rythme spatial semble être une constante, alors que le rythme temporel (vitesse de mise en place des entrenœuds) varie, surtout en fonction de la température. La réactivation de bourgeons axillaires du Ginkgo se produit quant à elle selon un rythme temporel, lors de l'affaiblissement de l'allongement caulinaire et selon un rythme spatial, puisqu'elle est localisée au niveau des entrenœuds longs. La succession d'entrenœuds longs et courts constatée sur les auxiblastes est identique à celle relevée sur la deuxième et la troisième pousses de l'axe principal (résultats non présentés).

Le déterminisme de la croissance rythmique a suscité de nombreuses hypothèses. Pour certains auteurs, les jeunes feuilles sont en grande partie responsables du fonctionnement rythmique de l'apex. La mise en évidence de reprises d'activité ou d'une croissance caulinaire permanente chez une espèce à croissance habituellement rythmique, après ablation des feuilles, plaide en faveur de l'intervention d'un tel mécanisme (Scarrone, 1964; Mialoundama, 1979, 1980; Mialoundama et al, 1984). L'ablation de feuilles de taille variable permet d'envisager un ensemble plus complexe de "corrélations à très courtes distances" s'exerçant entre "territoires de l'extrêmité de la pousse en croissance : apex, tissus sous-jacents au bourgeon terminal, jeunes feuilles" (Barnola et al, 1986). Champagnat et al (1986) distinguent 3 types de corrélations dépen- 
dantes de la taille des jeunes feuilles du Chêne et s'exerçant successivement : corrélation jeune feuille $(<5 \mathrm{~mm})$ - apex, corrélation feuille $(13-20 \mathrm{~mm})$ - jeune feuille, corrélation feuille (> $20 \mathrm{~mm}$ ) - tige.

Pour expliquer le pouvoir d'inhibition des jeunes feuilles en croissance sur le méristème apical, Hallé et Martin (1968) avaient suggéré l'existence d'une compétition vis-à-vis de l'eau entre ces 2 territoires. Celle-ci cesserait lorsque les feuilles devenues adultes et moins avides d'eau permettraient de nouveau l'alimentation hydrique du méristème. Chez le chêne, les résultats de Alatou et al (1989) ne confirment pas cette hypothèse, mais mettent en évidence les capacités de puits des jeunes feuilles. Celles-ci accumulent les assimilats au détriment du méristème apical, qui était le puits principal au début de l'étalement des feuilles et de l'entrée d'eau dans ces organes. Au cours de l'allongement, la concentration en métabolites des feuilles diminue et la corrélation feuille-apex s'affaiblit. Une corrélation feuille-feuille qui est responsable de l'hétéroblastie, autre constante de la croissance rythmique chez le chêne s'établit (Champagnat et al, 1986).

L'allongement rythmique des entrencuds et leurs longueurs finales variables sont en partie sous contrôle des feuilles (corrélation feuille-tige). Maillard et al (1987b) ont démontré l'influence des feuilles sur les entrenceuds situés juste audessous et au-dessus d'elles. L'ablation d'une très jeune feuille stimule l'allongement de l'entrenceud sous-jacent et limite celui de l'entrenœud sus-jacent. De tels traitements peuvent donc permettre de transformer des entrenœuds longs en entrenœuds courts et vice-versa, modifiant ainsi la structure caractéristique de l'axe principal pour une espèce végétale donnée. Pour Millet (1968, 1970), l'origine de l'alternance rythmique de longueur des en- trencuds de la fève pourrait se situer dans l'activité de mérèse variable de l'apex, qui attribuerait de façon périodique un plus ou moins grand nombre de cellules à chaque entrenœud.

Outre les corrélations à courte distance, certains auteurs suggèrent l'existence possible de corrélations à longue distance entre le méristème terminal et les racines. Ainsi, chez le mandarinier, la croissance rythmique pourrait être expliquée par des variations périodiques de quantité de nutriments disponibles, liées à l'absorption variable d'un système racinaire doué également d'une croissance rythmique (ElMorsy et Millet, 1989). Pour Borchert (1973), la croissance de la tige étant beaucoup plus rapide que celle des racines, l'existence d'une croissance rythmique limiterait le développement de la tige et maintiendrait un rapport croissance racinaire/ croissance caulinaire constant.

En conclusion, l'existence d'un rythme de fonctionnement propre au méristème apical du Ginkgo biloba est mis en évidence par une culture en conditions contrôlées. II n'a pas été possible d'effectuer l'analyse mathématique de ce rythme de croissance, car les conditions choisies n'assurent pas une croissance prolongée d'une majorité des plants. Des conditions de températures élevées et constantes associées à des éclairements forts permettraient sans doute cette étude. II nous semblerait également intéressant de tenter de modifier l'architecture du Ginkgo et l'évolution des unités morphogénétiques au moyen de suppressions de feuilles ou du bourgeon terminal réalisées en conditions contrôlées. Par ailleurs, il serait important de préciser la nature du repos dans lequel se trouve le bourgeon terminal entre 2 périodes de croissance active : des traitements par le froid apporteraient sans doute les premiers éléments de réponse. 


\section{REMERCIEMENTS}

Ce travail a été réalisé au Phytotron (CNRS, Gif/Yvette), dans le cadre d'une convention CIFRE (convention industrielle de formation par la recherche) établie entre le laboratoire Beaufour, le laboratoire de physiologie du développement des plantes, Université Paris VI et le laboratoire de botanique et de phytochimie, Universié Paris XI.

\section{RÉFÉRENCES}

Alatou D, Barnola P, Lavarenne S, Gendraud M (1989) Caractérisation de la croissance rythmique du Chêne pédonculé. Plant Physiol Biochem 27, 275-280

Alvim PT, Alvim R (1976) Relation of climate to growth periodicity in tropical trees. In: Tropical trees as living systems (Tomlinson PB, Zimmerman $M H$, eds) Cambridge Univ Press, 445-464

Barnola P, Champagnat P, Lavarenne S (1977) Mise en évidence d'une dormance rythmique chez le Noisetier (Corylus avellana L) cultivé en conditions contrôlées. $C A$ Séances Acad Sci Paris, sér D 284, 745-748

Barnola P, Crochet A, Payan E, Gendraud M, Lavarenne S (1986) Modifications du métabolisme énergétique et de la perméabilité dans le bourgeon apical et l'axe sous-jacent au cours de l'arrêt de croissance momentané de jeunes plants de Chêne. Physiol Vég 24, 307-314

Borchert R (1973) Simulation of rythmic tree growth under constant conditions. Physiol Plant 29, 173-180

Champagnat $P$, Payan $E$, Champagnat $M$, Barnola P, Lavarenne S, Bertholon C (1986) La croissance rythmique de jeunes Chênes pédonculés cultivés en conditions contrôlées et uniformes. Coll Int Arbre, Naturalia Monspeliensia, 303-337

Critchfield WB (1970) Shoot growth and heterophylly in Ginkgo biloba. Bot Gaz 131, 150162

De Bilderling N, Lourtioux A (1976) Quinze années de phytotronique. In: Études de Biologie Végétale. Hommage au Professeur
Pierre Chouard (Jacques R, éd) Paris, 331341

De Sloover J (1961a) Quiescence estivale et dormance chez Ginkgo biloba L. Bull Acad Roy Belg Cl Sci Ser 5 XLVIII, 459-465

De Sloover J (1961b) Effets de la lumière sur la croissance juvénile de Ginkgo biloba L. I. Étude d'une population de semis. Bull Jard Bot Etat Brux XXXI, 481-506

De Sloover J (1962) Effets de la lumière sur la croissance juvénile de Ginkgo biloba L. II. Autoécologie expérimentale. Bull Jard Bot Etat BruX XXXII, 29-86

El-Morsy AA, Millet B (1989) Analyse de la croissance rythmique du Mandarinier commun (Citrus deliciosa Tenore) cultivé en conditions constantes. Fruits 44, 21-27

Gunckel JE, Thimann KV (1949) Studies of development in long shoots and short shoots of Ginkgo biloba L. III. Auxin production in shoot growth. Am J Bot 36, 145-151

Hallé F, Martin R (1968) Étude de la croissance rythmique chez l'Hévéa (Hevea brasiliensis Mull Arg Euphorbiacées-Crotonoidées). Adansonia 8, 475-503

Juntilla $O$ (1980) Effect of photoperiod and temperature on apical growth cessation in two ecotypes of Salix and Betula. Physiol Plant 48, 347-352

Lavarenne-Allary S (1965) Recherche sur la croissance des bourgeons du Chêne et de quelques autres espèces ligneuses. Ann Sci For 22, 1-203

Lavarenne S, Champagnat $P$, Barnola $P$ (1971) Croissance rythmique de quelques végétaux ligneux de régions tempérées cultivés en chambres climatisées à température élevée et constante et sous diverses photopériodes. Bull Soc Bot Fr 118, 131-162

Maillard $P$, Jacques $M$, Miginiac $E$, Jacques $A$ (1987a) Croissance de jeunes Terminalia superba en conditions contrôlées. Ann Sci For 44, 67-84

Maillard P, Jacques M, Miginiac E (1987b) Correlative growth in young Terminalia superba in controlled environment: effect of the leaves on internode elongation. Ann Bot 60, 447454

Mialoundama F (1979) Influence des feuilles sur la croissance en longueur de la tige chez 
Gnetum africanum Welv. $C A$ Séances Acad Sci Paris, Sér D 288, 603-606

Mialoundama $F$ (1980) Action régulatrice des feuilles sur l'activité morphogénétique du bourgeon terminal chez Gnetum africanum Welw. C R Acad Sci Paris, Sér D 291, 509512

Mialoundama F, Lauzac M, Paulet P (1984) The periodic induction of dormancy during the rythmic growth of Gnetum africanum. Physiol Plant 61, 309-313

Millet $B$ (1968) Action régulatrice d'une ablation de feuilles sur l'alternance de longueur des entrenœuds chez la Fève (Vicia faba L). $C R$ Séances Acad Sci Paris, Sér D 266, 1956 1958

Millet B (1970) Analyse des rythmes de croissance de la Fève Vicia faba $L$. Thèse Doct Sci, Univ Besançon, 132 p

Morgan DC, Warrington IJ, Halligan EA (1985) Effect of temperature and photosynthetic photon flux density on vegetative growth of
Kiwi fruit (Actinidia chinensis). $N Z J$ Agric Res 28, 109-116

Nozeran R, Demni S, Bouzid S, RossignolBancilhon L (1983) Analyse du comportement morphogénétique de jeunes Bigaradiers (Citrus aurantium L, Rutacées). Bull Soc Bot Fr, Lett Bot 130, 109-129

Scarrone F (1964) Pouvoir inhibiteur des feuilles de grande taille chez le Manguier (Mangifera indica L). C R Séances Acad Sci Paris, Sér D $259,4342-4345$

Scarrone F (1965) Rôle respectif des rythmes endogènes et des facteurs climatiques dans la croissance du Manguier (Mangifera indica L). C R Seances Acad Sci Paris, sér D 260, 3469-3472

Turner DW, Lahav E (1983) The growth of Banana plants in relation to temperature. Aust $J$ Plant Physiol 10, 43-53

Warrington IJ, Edge EA, Green LM (1978) Plant growth under high radiant energy fluxes. Ann Bot 42, 1305-1313 